\title{
Building potential for graphene photodetectors
}

\author{
Nathan O Weiss ${ }^{1}$ and Xiangfeng Duan ${ }^{2,3}$ \\ NPG Asia Materials (2013) 5, e74; doi:10.1038/am.2013.64; published online 6 December 2013
}

Photodetectors are ubiquitous, found in everyday products such as TV remotes, disc players and digital cameras as well as specialized devices for fiber optic communications and astronomical observations. Similarly, graphene has seen a quick emergence in a range of prototypic devices due to its attractive electronic, optical and mechanical properties. ${ }^{1}$ Using graphene in photodetection utilizes its high carrier mobility and zero bandgap that-among other advantages—-show promise for wide-spectrum, high-speed, low-cost and flexible photosensors. Graphene-based photodetectors have typically relied on Schottky barriers formed near the metal-graphene contacts, where a built-in potential drives the separation and transport of photogenerated electron-hole pairs. However, symmetric metal-graphene-metal devices generate an equal positive and negative flow with a net zero photocurrent (Figure 1a). Using metals with asymmetric band structures breaks this equilibrium ${ }^{2}$ (Figure 1b) at the cost of additional fabrication steps and is limited by the maximum difference in barrier heights. Recently, Liu and co-workers ${ }^{3}$ from Peking University presented a step forward by creating a single $\mathrm{p}-\mathrm{n}$ junction in graphene itself.

In this study, adjacent regions of intrinsic (p-type) and nitrogen-doped (n-type) graphene form a junction with a potential offset at the center of the device. With only one junction, a unidirectional carrier separation and transport produces a measurable photocurrent (Figure 1c). The synthesis is built upon their previous work on 'mosaic graphene':4 by modulating the doping concentrations during a chemical vapor deposition process, they form coherent regions of distinctly nitrogen-rich (doped) and -deficient (intrinsic) graphene. Substitutional doping with nitrogen shifts the Fermi level and forms a $\mathrm{p}-\mathrm{n}$ junction at the interface that operates independently of any contact metals.

\footnotetext{
${ }^{1}$ Department of Materials Science and Engineering, University of California, Los Angeles, CA, USA; ${ }^{2}$ Department of Chemistry and Biochemistry, University of California, Los Angeles, CA, USA and ${ }^{3}$ California NanoSystems Institute, University of California, Los Angeles, CA, USA

E-mail: xduan@chem.ucla.edu
}

Less than $1 \mathrm{~nm}$ thick, a single layer of graphene absorbs $2.3 \%$ of the light that passes through: extraordinarily opaque for an atomic monolayer, yet still highly transparent. While low absorption is great for transparent conductors, in photodetectors it leads to low photoresponsivity. Gold
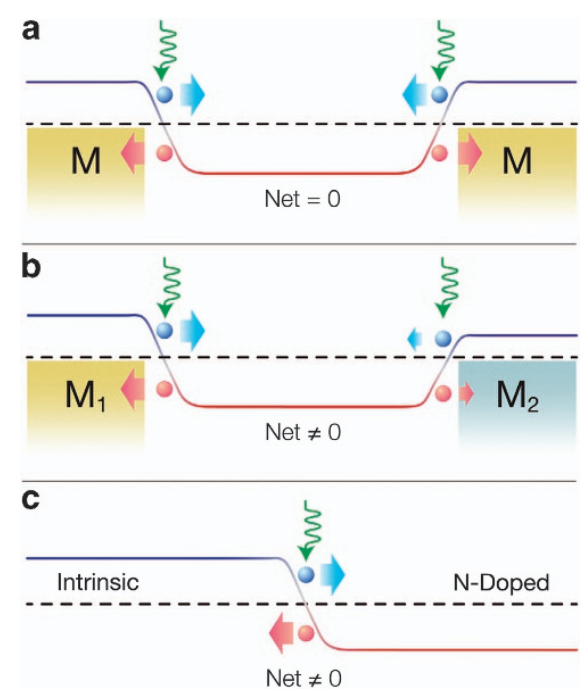

Figure 1 Graphene-based photodetectors. (a, b) Band diagrams for symmetric (a) and asymmetric (b) graphene-metal Schottky junction-based photodetectors with a zero and non-zero photocurrent, respectively. Contacting graphene with metals of variable work function changes its Fermi level and creates a potential offset near graphene-metal contact that facilitate the separation and transport of photocarriers. (c) Band diagram of a graphene $p-n$ junction-based photodetector with a net photocurrent. The potential offset near the $p-n$ junction can effectively drive the separation and transport of photocarriers to produce a measurable photocurrent. Dotted lines are the Fermi level position and solid blue-red lines are the positions of the Dirac point in graphene. Blue and red spheres represent electrons and holes, respectively. nanoparticles are used as nanoscale optical antennae that concentrate the light through plasmonic effects, ${ }^{5}$ enhancing the absorption, photocurrent and photosensitivity of the graphene $\mathrm{p}-\mathrm{n}$ junction.

These photodetectors could benefit a range of optoelectronic devices that require high photoresponsivity, flexibility and wavelength selectivity. Doped-graphene $\mathrm{p}-\mathrm{n}$ junctions offer more practical and self-contained devices over graphenemetal junctions, though much work remains before the quality and yield of either can compete with state-of-the-art silicon-based detectors. Nevertheless, this synthesis and fabrication of graphene $p-n$ junctions with integrated plasmonic antennae provide a novel platform for photodetection that utilizes the low absorption of atomically thin materials and offers unique opportunities for engineering new types of photovoltaic and optoelectronic devices.

1 Weiss, N. O., Zhou, H., Liao, L., Liu, Y., Jiang, S., Huang, Y. \& Duan, X. Graphene: an emerging electronic material. Adv. Mater. 24, 5776 (2012).

2 Mueller, T., Xia, F. \& Avouris, P. Graphene photodetectors for high-speed optical communications. Nat. Photon. 4, 297-301 (2010).

3 Wu, D., Yan, K., Zhou, Y., Wang, H., Lin, L., Peng, H. \& Liu, Z. Plasmon enhanced photothermoelectric conversion in chemical vapor deposited graphene pn junctions. J. Am. Chem. Soc. 135, 10926-10929 (2013).

4 Yan, K., Wu, D., Peng, H., Jin, L., Fu, Q., Bao, X. \& Liu, Z. Modulation-doped growth of mosaic graphene with single-crystalline $p-n$ junctions for efficient photocurrent generation. Nat. Commun. 3, 1280 (2012).

5 Liu, Y., Cheng, R., Liao, L., Zhou, H., Bai, J., Liu, G., Liu, L., Huang, Y. \& Duan, X. Plasmon resonance enhanced multicolour photodetection by graphene. Nat. Commun. 2, 579 (2011).

\footnotetext{
cc) (i) $($ ) $\odot$ This work is licensed under a Creative Commons AttributionNonCommercial-NoDerivs 3.0 Unported License. To view a copy of this license, visit http://creativecommons.org/licenses/bync-nd/3.0/
} 